\title{
ESPACIO MODERNO: \\ FUNCIONALISMO \\ REDUCCIONISTA
}

J. PABLO MONTES LAMAS 


\section{J. PABLO MONTES LAMAS}

Arquitecto. Maestro en arquitectura por la Universidad Nacional Autónoma de México. Doctorante en arquitectura por la unAm con la tesis de investigación «Arquitectura experimental, del cinismo al quinismo». Su área de investigación es la arquitectura experimental. Actividad profesional independiente. 


\section{RESUMEN \\ ABSTRACT}

En cuanto a movilidad humana en el espacio urbanoarquitectónico, la Modernidad representó la entronización de lo inmóvil y lo comprimido por medio de formas de control practicadas en el taxativo espacio moderno. Ello condujo al planteamiento de la composición arquitectónica desde un sistema cartesiano, cerrado y disciplinar. Las formas pedagógicas, constructivas y de diseño en arquitectura y urbanismo modernos estuvieron influenciadas decisivamente por un régimen que en el pensamiento occidental apuntó a domesticar el cuerpo y controlar el espacio. La Posmodernidad, como oposición cultural, quiere emanciparse de estas restricciones y con ello pone en marcha un proceso de insurrección. Este artículo trata de las formas disciplinares ilustradas que dieron consistencia al espacio moderno, cuyas representaciones geométricas fueron el plano y el cuadro -con sus respectivas enunciaciones dialécticas-, de las lógicas latentes de la llustración que tienen como consecuencia un espacio disciplinar, y de las características que lo definen en oposición a los rasgos espaciales (traducidos a modelos) de la Posmodernidad: la ruptura del idealismo platónico, la transgresión de las contraculturas, el redescubrimiento de lo extenso en oposición a un espacio teórico y compacto y su entendimiento no dialéctico sino (en sentido deleuziano) rizomático y desplegado.

Palabras clave: espacio moderno, control, cinismo, transgresión, funcionalismo, posmodernidad, crítica, contracultura.

In terms of human mobility in urban-architectural space, Modernity represented the enthronement of the immovable and the compressed through methods of control exercised in the punitive modern space. This led to the approach of the architectural composition from a cartesian, closed and disciplinary system. Teaching, building and design in modern architecture and urbanism were decisively influenced by a regime that in western thought aimed to tame the body and control the space. Postmodernity as cultural opposition attempts to emancipate itself from these restrictions and thus, start a process of uprising. This article is about disciplinary patterns from the age of Enlightenment which gave consistency to the modern architectural space whose geometric representations are the plane and the square -with their respective dialectical expressions-, the latent logic of the Enlightenment which resulted in a disciplinary system, and its defining characteristics, as opposed to spatial features (translated into models) of Postmodern age: the breakdown of platonic idealism, the transgression of the countercultures, the rediscovery of the extended as opposed to theoretician and compact space, and its non-dialectical understanding but (in deleuzian sense) rhizomatic and unfolded.

Keywords: Modern space, control, cynicism, transgression, functionalism, postmodernity, critic, counterculture. 
Ahora ya sabemos que el alma es el cuerpo, y el cuerpo el alma. Nos dicen que son diferentes porque quieren persuadirnos de que podemos quedarnos con nuestras almas si los dejamos esclavizar nuestros cuerpos.

George Bernard Shaw

 a concepción moderna del espacio se empieza a fraguar a partir de la segunda globalización, que de acuerdo con Sloterdijk se da con la exploración marítima y la circunnavegación de la tierra por Magallanes. Este desarrollo agudiza la concepción del territorio y el espacio, que desde una obsesión cartográfica y colonialista, es reducido a su mínima expresión, a un espacio teórico y comprimido que consuela al habitante de la tierra (lo seco) frente al horror del vacío "blanco», el vacío de los mapas y la inhabitabilidad de los mares. El espacio moderno deviene teórico y comprimido y se convierte, gracias a la arquitectura y el urbanismo, en una herramienta eficaz de disciplina y disuasión. Los modelos derivados de este espacio geográfico fungen como prototipos de la composición que los reproduce vehementemente en la ciudad; son elementos generadores de una arquitectura de control plasmados en lo urbanoarquitectónico. Esta es una de las grandes estrategias del funcionalismo para introducir en el proyecto, desde una postura dialéctica, el racionalismo, el positivismo y el argumento ilustrado, y de este modo instaurar una arquitectura jerárquica, centralista, idealista-platónica y, sobre todo, de control, que viene a emanciparse de estas condiciones en la Posmodernidad, con una actitud cínica, o mejor, quínica.
Antes de continuar, se precisan ciertas consideraciones. El cinismo del que se habla en este artículo, a pesar de que derivado de las descalificaciones del proceso ilustrado y en el sentido usual del término ostenta connotaciones negativas, se toma siempre en sentido positivo, desde Sloterdijk y Foucault. Luego, cuando se habla del estudio del espacio, no se reniega de su condición extensa en función de la teorización, es más, en lo urbanoarquitectónico se entiende como contraposición del «espacio cerrado» delimitado por elementos arquitectónicos y esencialmente idealista, ya que el idealismo, hijo del platonismo, pertenece a una conciencia histórica occidental que no se pudo y tampoco se quiso erradicar. Una tercera y última consideración es sobre el término «urbanoarquitectónico»; no es lo urbano visto desde lo arquitectónico ni la arquitectura desde lo urbano, no es una interdisciplina, sino una transdisciplina, o mejor, una multiplicidad. El término es un concepto original creado por Farías (2003) a partir del discurso libre indirecto y la estrategia deconstructiva. Es una forma sumamente contemporánea de entender el espacio, desde la óptica de Deleuze y Guattari, Derrida y Koolhaas.

Ahora bien, si es cierto que el espacio moderno era socialmente orientado y que el funcionalismo era una táctica (no de control, sino) de legitimación de la función social, o sea, de la interacción del ser humano con el espacio, ¿por qué la arquitectura y el urbanismo devinieron espacios de representación? La suposición hipotética es que el funcionalismo en realidad fue el pretexto para la imposición de las formas de control en el espacio social. El hombre desde la prehistoria se enfrenta al infinito y surgen preguntas antropológicas 
y existenciales, y al intentar darles respuesta ha generado una compulsión por nombrar, esto es, de definir, controlar, delimitar, para dar sentido y desde ahí aprehender los conceptos. Por ello el espacio, no exento de este tratamiento, también fue sistematizado y retratado en modelos que permitieran su comprensión; éstos han evolucionado a través del tiempo de forma acumulativa, trasluciendo y evidenciando la idea que se tiene de él; la concepción y entendimiento de los arquetipos se enfrentan a un proceso evolutivo y acumulativo como en una sucesión de estratos, en tanto que posee huellas de distintas sociedades que lo organizaron en el proceso histórico. Por tal motivo, se precisa del análisis de las formas en que es percibido y de la evolución de sus representaciones. Como resultado de esta comprensión y abstracción espaciales que indudablemente han influido a los arquitectos de cada época, ellos han planteado la arquitectura basada en los arquetipos que de él se tienen; su desarrollo es particularmente claro.

\section{DEL ESPACIO ANTIGUO...}

Es posible rastrear a través de los elementos genéticos del lenguaje (y luego en los procesos históricos) las concepciones que se tienen del espacio antiguo. Al hacer uso de la raíz etimológica, en la propia palabra se encuentra inserto un primer modelo: proviene de la locución latina spatium, vocablo que señala un tiempo de espera entre dos puntos temporales, y se aplica a una distancia vacía entre dos puntos. En el siglo xIx, Mommsen, Ross y El diccionario etimológico del latín creían que la palabra provenía del griego spodium, que se traduce como campo de carreras o cualquier lugar apto para caminar. Después esta versión fue rebatida y descartada. Roberts y Pastor luego afirman que spatium procede de la raíz indoeuropea spe- (expandirse, prosperar), y Pokorni, a su vez, la relaciona con $s p(h) e^{-} i$ - /spi- sphe - y que es la misma que en latín es spes (esperanza), de donde se vinculan esperanza y esperar, así como esfera.

Esta primera definición-modelo, aunque conserva su estructura semántica, al pasar por el tamiz del tiempo y la cultura ha dejado de ser útil para la comprensión del espacio debido a que hoy se asocia a otro concepto: «línea», o sea distancia entre dos puntos. Pero, ¿cómo es que la idea de línea llega a ser «espacio» en el sentido moderno a través de un proceso de desplazamiento geométrico y desdoblamiento mental? ¿Cómo se evoluciona desde los dos puntos teóricos, del principio y fin, del $a$ y $b$ al plano y luego a un despliege multidimensional?

La idea que se tiene del espacio, como distancia entre dos puntos, es particularmente clara en los filósofos griegos; cuando tratan de explicarlo recurren a un modelo de distancia interpuntual, como en las demostraciones de Zenón de Elea y sus discípulos. La tortuga y Aquiles sólo se desplazan a través de momentos (átomos), de instantes puntuales en el tiempo. De ahí el vínculo con su definición etimológica que incorpora en todo momento al tiempo, condición que le es inherente. Pero urbana y arquitectónicamente, las implicaciones de esta representación espacial se plasman en los proyectos arquitectónicos de la época, por ejemplo en la Acrópolis de Atenas, que conecta puntos en una trayectoria «cinematográfica». ${ }^{1}$

1. Cf. Farías, 2003 
El espacio de la antigüedad en su forma abstracta puede ser leído a través de la conexión de puntos por medio de distancias vacías. Este vacío es teórico y no físico, desde luego, y puede interpretarse como la carencia de significación y de contenido, esto es: no hay elementos materiales a los que se pueda asociar un significado, ni suficiente cohesión de contenidos que permitan coligar elementos metafísicos a este espacio interpuntual. Sin embargo, estas distancias «vacías» o indeterminadas (de escala humana) están supeditadas a un gran recipiente, una esfera omniterritorial, pura y perfecta, que es el continente del todo (de escala cósmica). Este gran espacio infinito es lo que Platón Ilama la Chora, el espacio antiguo, indivisible, «extenso en toda su extensión», aunque no necesariamente infinito. Esta representación, innegablemente antropocéntrica, dominó en el pensamiento antiguo y sirvió como principio modélico de su arquitectura. El gran espacio (que hacia el siglo xx se extendió a escalas astronómicas) se deduce entonces como una esfera omnicomprensiva que encierra una abstracción de él mismo, que es social y de escala humana plasmada en el desarrollo de la arquitectura. De este modo, hay una coexistencia entre dos formas de pensar el espacio que elementalmente se traducen como la esfera y la línea.
La cosmología de la antigüedad occidental, sobre todo la platónica y la de los sabios helenísticos posteriores, se había adscrito a la idea de representar la totalidad de lo que es mediante la imagen estimulante de una esfera omnicomprensiva. [...] Al mismo objeto se le llamó a la vez cielo, uranos. El nombre titánico expresaba la idea de que el mundo tiene su límite en una última bóveda de éter: una conjetura que hubiera podido Ilamarse igualmente una esperanza. Gustaba de imaginarse el mundo como una amplia vasija, que proporciona sostén a las estrellas fijas y calma el miedo humano a caer. El cielo era para Aristóteles la última envoltura, que todo lo contiene pero no es contenida por nada. Mensurar ese cielo en pensamientos significó llevar a cabo la primera globalización. Con ello surgió la buena nueva de la filosofía: que, por mucho que le deprima el desorden en que vive, el ser humano no puede caerse fuera del universo. ${ }^{2}$

Estas categorías espaciales dotadas de significación, y ya no como res extensa, adquirieron características sociales, relacionales e históricas de diferentes intensidades. Algunas llevaron esta significación a un grado inviolable y cuasi absoluto, que vinculado a la conexión trascendente dio origen al nacimiento del espacio sa-

2. Sloterdijk, 2005: 25 . 
01.

Última descripción de la Tierra entera.

Planisferio, 1602. Gerardo Mercatore .

"Domini est terra et plenitudo eius orbis terrarum et uni versi qui habitant in eo. Ouia super maria fundavit eum.»

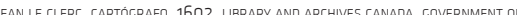
GANADA. DISPONBEE EN WEB:<HTTP://COHECTIONSCANADA GC.CA/>

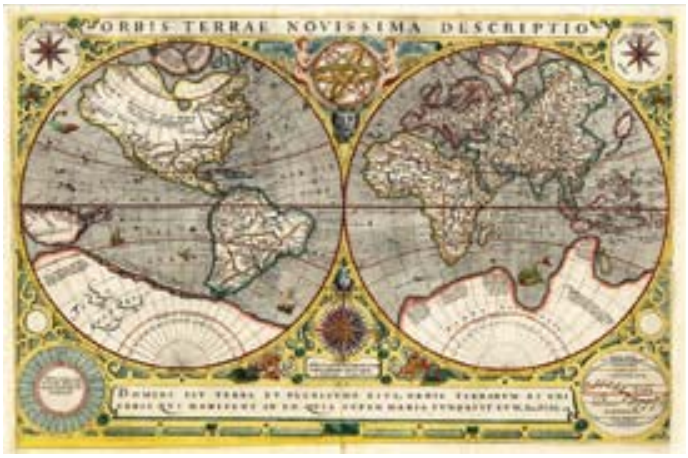

01

sión de un espacio planisférico en el que estaba implicada la definición -entendida como imposición del límite- tanto como espacio superficial-continente del punto y la línea, o sea el campo, como por la retícula de la representación sobre el papel.

Cuando los mapamundis planisféricos arrinconan el globo, cuando el mismo Atlas ya no aparece soportándolo sino que se presenta como libro de mapas encuadernado -esa transposición fue realizada por la colección de mapas más influyente de la Edad Moderna: Gerardi Mercatoris Atlas sive cosmographicae mediations de fabrica mundi et fabricati figura, Ámsterdam, 1608-1609-, entonces triunfa el medio bidimensional sobre el tridimensional e, ipso facto, la imagen sobre el cuerpo. ${ }^{4}$

En la Modernidad la relación interpuntual se despliega, se ensancha, se proyecta; ahora la línea está contenida en el plano a la vez que es su generatriz, como antes fue el punto respecto de la línea. ${ }^{5}$ Esta absorción de lalínea en el plano se representó en el modelo espacial de abscisas y ordenadas, quizá más claro para la aplicación en la arquitectura y el urbanismo; ciudades $x-y$, la en-

4. Sloterdijk, 2005: 126.

5. Como pone de manifiesto Leibniz, que luego toma Deleuze en $E$ I Pliegue (1989) 
tronización del cuadro. Ejemplos de ello, las ciudades novohispanas como principio de modernidad o el trazo de Nueva Ámsterdam que se redefine por Witt, Morris y Rutherford en 1807 como una retícula absoluta de 2.028 manzanas, ${ }^{6}$ estos prototipos se extendieron a la mayoría de las ciudades industriales, desplazando por mucho a los modelos premodernos de ciudades ideales. No sólo eso, también las formas de representación del proyecto siguieron estas directrices territoriales, del plano a la zonificación. El entendimiento del espacio como superficie planimétrica con las implicaciones de ordenamiento y control disciplinar que consecuentemente trajo consigo el modelo ilustrado.

Si bien este modelo organizativo de zonificación ya es aplicado en la antigua Grecia, y sobre todo en el Imperio Romano (los districtus), la ciudad moderna ya no está dividida por distancias vacías (de un pueblo a otro), sino de carácter administrativo, pero sobre todo disciplinar. La distancia física deviene ideológica y luego, al ser asimilada, también psíquica. ${ }^{7}$ Esta estrategia que tiene origen primero con la definición de la propiedad feudal, se intensifica en la sociedad industrial (aunque no se elimina completamente, porque a fin de cuentas todo pertenece al rey) y luego, de forma más clara, con la zonificación al interior de la demarcación de las ciudades, como la París de Haussmann.

6. Cf. Koolhaas, 1994

7. En la danza, como interacción del cuerpo con el espacio, es claro, según Au (2012): «La percepción de la audiencia respecto al bailarín comenzó a cambiar. [...] El proscenio creó distancia física y psíquica entre los artistas y los espectadores quienes terminaron por ya no identificarse con el bailarín».

\section{EL ESPACIO DE CONTROL}

Foucault, en Vigilar y castigar, estudia el arte de las distribuciones y cómo es que la sociedad disciplinaria y punitiva sistematiza el espacio en función de la instauración del orden. Se inventan a partir de este proceso mecanismos de restricción que fueron sumamente habituales en la arquitectura moderna, elementos de uso común tanto en la proyectación del espacio como en la concepción de la idea, tales como el plano, la retícula, y el funcionalismo.

La primera condición del arte de las distribuciones como estructura espacial de control es la clausura, herencia de un cinismo desviado en los monjes medievales y apéndice de las murallas. La forma de clausura es la concreción material de la noción de límite, que en la Modernidad evoluciona hacia la disuasión. Si en las edades Antigua y Media el límite es un elemento físico y material que define la clausura, la Modernidad logra establecer en el espacio de control límites imaginarios, coercitivos y disuasores. La clausura, el espacio cerrado, es también una reminiscencia de la esfera, del cuerpo total, puro, inerte y platónico en el que habita lo perfecto. En el caso de los conventos, por ejemplo, sólo en el interior habita lo sagrado, el exterior es fuente de imperfección.

Pero la clausura no es suficiente, porque es posible encadenar el cuerpo pero no el espíritu humano (esta es la lección aprendida por el conde de Montecristo de Dumas, «el lugar-habitar en la piel del otro no puede robarse ni alquilarse ${ }^{8}$ ), es necesario un sistema espa-

8. Sloterdijk, 2005: 304 
cial organizativo que controle al sujeto. Los aparatos disciplinarios, afirma Foucault, trabajan de una manera mucho más flexible y fina que bajo el principio de clausura, en primer lugar, según el principio de localización elemental o de la división en zonas. A cada individuo su lugar, y en cada emplazamiento un individuo. Evitar las distribuciones por grupos; descomponer las implantaciones colectivas; analizar las pluralidades confusas, masivas o huidizas. El espacio disciplinario tiende a dividirse en tantas parcelas como cuerpos o elementos haya para repartir. Es preciso anular los efectos de las distribuciones indecisas, la desaparición incontrolada de individuos, su circulación difusa, su coagulación inutilizable y peligrosa; táctica de antideserción, de antivagabundeo, de antiaglomeración. Se trata de establecer las presencias y las ausencias, de saber dónde y cómo encontrar a los individuos, de instaurar comunicaciones útiles, de interrumpir las que no lo son, de poder en cada instante vigilar la conducta de cada uno, apreciarla, sancionarla, medir las cualidades o los méritos. Procedimiento, pues, para conocer, para dominar y para utilizar. La disciplina goza de un espacio analítico. ${ }^{9}$

Esta división zonal es la principal característica organizativa de la ciudad moderna y de todos los planes de desarrollo y las estrategias de planeación de las políticas estatales. El espacio de la ciudad y la arquitectura (este proceso de zonificación no está exento del proyectar arquitectónico gracias a la histérica Modernidad) está sometido a regímenes de orden que fácilmente devienen espacios de vigilancia.

9. Foucault, 2013: 166
Una tercera condición del espacio de control, aun más próxima a la visión de la arquitectura moderna, es el funcionalismo, la estrategia de inserción del proyecto en la cultura, con lo que Foucault señala como «la cremallera del monstruo». Para el modernismo este es el principal argumento de autoridad para la implantación del proyecto. Desde luego, en un pensamiento dialéctico, su opuesto es la forma, subordinada siempre a la función, como afirmó Sullivan. Este pensamiento se deriva de las estrategias de control introducidas de antemano por la Ilustración en la cultura, y son persistentes como estatuto funcionalista en La Escuela de Chicago, la Bauhaus, y desde luego en las escuelas de pensamiento derivadas de Le Corbusier, Mies y Johnson, los idealistas más influyentes, el factor clave de la propagación del funcionalismo. Foucault le llama «la regla de los emplazamientos funcionales» y responde a la necesidad de crear un espacio útil. Este proceso arquitectónico aparece claramente en los hospitales (¿dónde, si no?) y se extiende a las fábricas de finales del siglo xvill; de este modo, el espacio de control da lugar a los sistemas de producción (en serie) en la medida que ésta se divide y el proceso de trabajo se articula de acuerdo a las fases y a los individuos, pero fundamentalmente al esquema jerárquico de un agente que supervisa la producción: «cada variable -vigor, rapidez, habilidad, constancia- puede ser observada, y por lo tanto, caracterizada, apreciada, contabilizada y referida a aquel que es su agente particular». En este sentido, espacio e individuo se ensamblan, como la conexión maquínica ${ }^{10}$ que puede establecer un obrero o un oficinista respecto de su espacio de trabajo.

10. En el sentido deleuziano. Deleuze y Guattari (2004). 


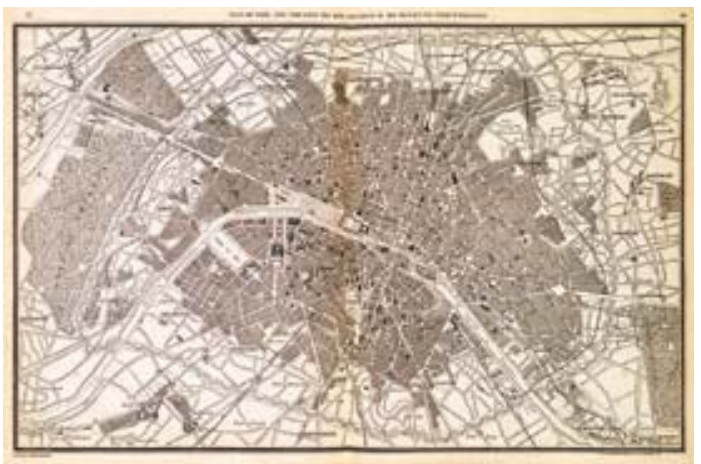

02.

Pero hay una cuarta condición del arte de las distribuciones en el sistema moderno de control, y es la interacción respecto de los individuos en el espacio, que en principio es esencialmente jerárquico (muchas veces piramidal) para activar la vigilancia. «Al organizar las "celdas", los "lugares" y los "rangos", las disciplinas fabrican espacios complejos: arquitectónicos, funcionales y jerárquicos al mismo tiempo.»11 Estas formas de situación del individuo en el espacio convergen hacia un punto, o mejor, hacia una abstracción espacial concreta formada de cuatro puntos: el cuadrado. Esta figura geométrica derivada del espacio cartesiano sintetiza en su esencia la estrategia proyectual y disciplinar de la Modernidad, aplicada de modo intensivo tanto en la planeación de las ciudades como en los formatos de proyectos, en las retículas funcionales y los esquemas estéticos que renunciaron abiertamente al ornamento.

El cuadro en el siglo xVIII es a la vez una técnica de poder y un procedimiento de saber. Se trata de organizar lo múltiple, de procurarse un instrumento para recorrerlo y dominarlo, de imponerle un «orden». [...] En tanto distribución disciplinaria, la ordenación en cuadro tiene como función tratar la multiplicidad por sí misma, distribuirla y obtener de ella el mayor número de efectos posibles. ${ }^{12}$

11. Foucault, 2013: 168.

12. Ibid, p. 173 .
02.

Plano de París, con indicaciones de las calles nuevas y los trabajos en curso de ejecución. Haussmann. 1853.

DELAMARE, F. CARTÓCRAFO, 1853. BROWN UNIVERSITY. DISPONIBLE EN WEB: <HTTP://LIBRARY.BROWN.EDU/ FIND/RECORD/DC1223576065671875>

\section{APOTEOSIS DEL LÍMITE}

Espacialmente, la Modernidad es la apoteosis del límite, de la barrera y el muro a través de la búsqueda de la contención del espacio que ya no es sólo físico sino mental. Las restricciones tampoco son necesariamente materiales sino disuasivas: el gran triunfo de la Modernidad en la arquitectura fue el límite inmaterial. A escala urbana, la zonificación en la ciudad dibuja perímetros administrativos, y en su proyectación resulta indispensable la construcción de límites imaginarios, aperturas contenciosas, amplitudes disuasivas bajo el pretexto de la eficiencia militar, fundando - siglos antes del fascismo- una arquitectura totalitarista reglada por el principio racional de la llustración de «saber es poder».

El tratamiento que brindan el pensamiento y la arquitectura modernos en términos generales al espacio es el de la negación dialéctica de sí mismo, de acuerdo a la cual debe existir un opuesto, una contraparte capaz de justificar la esencia primera. Cuando Hegel publica en 1808 Fenomenología del espíritu, se cocinaba un gran cambio en la sociedad que parecía ponerse en movimiento por la intervención del capitalismo temprano. La ciudad industrial está en auge y el filósofo se plantea ¿cómo entender racionalmente que una cosa puede cambiar de apariencia y seguir siendo la misma cosa? Decididamente el mo- 
vimiento industrial comporta otros procesos y principios de comprensión del espacio que acontecía; pero ¿cómo entender este espacio «infinito» que a la vez podía contenerse por medio de los elementos materiales de la arquitectura? La lógica de esta pregunta se propaga a través de la crítica del arte de Alois Riegl, quien sitúa como «esencia» de la arquitectura el concepto de espacio, que hasta entonces no había sido utilizado de manera explícita, y presenta como paradigma el interior delimitado y perfecto del Panteón de Roma. ${ }^{13}$

La respuesta provino de la misma fuente con la que Hegel explicó la sociedad, herramienta que luego se propagó indiscriminadamente para intentar dar respuesta a todo: la dialéctica de los opuestos, que en la filosofía moderna ya no estaba circunscrita al ámbito de la antigua retórica. Se presentan en el campo de batalla dialéctico «dos» nuevos adversarios: el espacio contenido versus su archinémesis, el espacio infinito. El espacio encuentra a su gemelo maligno en el «antiespacio», concepto generado para justificar una dialéctica superficial que -aunque no explora todavía los conceptos de antimateria- pretende en el espacio geográfico llamar a la existencia un fenómeno que a todas luces no existe, pero que es capaz de justificar

13. Cf. Montaner, 2011: 29 lógicamente ciertas incoherencias urbanas y arquitectónicas megalómanas.

Riegl, al presentar como paradigma del espacio al interior delimitado del Panteón, establece que es éste el espacio, pero ¿cómo justificar al elefante en la habitación? ¿Cómo explicar la presencia incómoda e ineludible del espacio genérico e infinito? Las vanguardias de finales del xvIII y principios del xx vuelven sobre el espacio extenso e incontenible, a través de su concepción como libre, fluido, ligero, continuo, abierto, infinito, secularizado, transparente, abstracto, indiferenciado y newtoniano, que se contrapone totalmente al espacio tradicional que es diferenciado volumétricamente, de forma identificable, discontinuo, delimitado, específico cartesiano y estático.

A esta nueva modalidad de espacio unos la denominaron «espacio-tiempo» en relación con la teoría de la relatividad de Albert Einstein y a la introducción de la variable del movimiento, y otros la calificaron como «antiespacio», por generarse como contraposición y disolución del tradicional espacio cerrado, delimitado por muros. ${ }^{14}$

La influencia del pensamiento dialéctico no estaba sólo en la filosofía o en la vida práctica, no se podía

14. Steven, 1980 
concebir sin encontrar su desarrollo también en la arquitectura a través de la negación espacial, un concepto tan insólito que rechazaba por oposición el infinito reduciéndolo conceptualmente ad absurdum. Se condensa este pensamiento a la negación del origen, de donde parte todo elemento material e inmaterial de la arquitectura y la ciudad: la extensión, la localización, y la materia. El espacio sólo era válido en la medida de su contención por medio de los elementos materiales del edificio. Esta forma de pensar generalizada trajo consecuencias en la forma en que se proyectó la ciudad; si no se piensa el espacio como continuo, como interacción global, como fluido, abierto e infinito, consecuentemente la forma de proyectar estará (y estuvo, y está) basada en proyectos-isla, diseñados a partir de una delimitación planisférica en principio, que no involucra conexiones con el continuo de la materia, el espacio y las interacciones sociales.

En este proceso se trata de comprender los procesos mediante los cuales la ciudad no es entendida como un todo, sino como una suma de partes, de islas, o sea, inconexa. Esta es la crítica que hace Koolhaas tanto en Prisioneros voluntarios de la arquitectura como en el apéndice de Delirious New York, y que es recurrente en su filosofía. En «La ciudad del globo cautivo» cada parcela está identificada con el pensamiento (cada ciencia, cada manía, el arte, la poesía o los tipos de locura), que puede traducirse aislado, de tal suerte que la ciudad es un archipiélago, más que un conjunto conectado: «En cada parcela se alza un basamento idéntico [...]. Para facilitar y fomentar la actividad especulativa, estos basamentos -verdaderos laboratorios ideológicos- están equipados para suspender leyes inoportunas y verdades irrefutables, y para crear condiciones físicas inexistentes». ${ }^{15}$

No sólo cada manzana delimitada por la retícula, sino cada parcela, es una isla de ciudad. La propiedad privada se lo ha tomado muy en serio, institucionalizando el régimen del control a través de los límites arquitectónicos. La gran mayoría de las obras de arquitectura representan un intento megalómano de imponer, no a través de una participación del espacio, sino, casi como de esferas urbanas, autosuficientes e independientes, desde luego no la autarquía ni la independencia derivada del pensamiento cínico, porque, como se ha dicho, a muchos niveles estas formas de «independencia» resultaron un régimen de esclavitud sufragánea, codependientes y depredadoras y más bien una suerte de guetos unidos por convenientia. ${ }^{16}$

El modelo espacial derivado de esta situación es el del reduccionismo y la abreviación de las transiciones (no sólo al «interior» de la arquitectura o el «exterior» urbano, sino en lo neurálgico de lo urbano-arquitectónico), un modelo segregacionista del espacio, una anulación selectiva y accidentada de territorios reales para funda-

\footnotetext{
15. Koolhaas, 1994: 295.

16. En el sentido que le da Foucault (1968): «la convenientia. A deci verdad, la vecindad de los lugares se encuentra designada con más fuerza por esta palabra que la similitud. Son «convenientes» las cosas que, acercándose una a otra, se unen, sus bordes se tocan, sus franjas se mezclan, la extremidad de una traza el principio de la otra. Así, se comunica el movimiento, las influencias y las pasiones, lo mismo que las propiedades. De manera que aparece una semejanza en esta bisagra de las cosas. Doble desde que se trata de aclararla: semejanza del lugar, del sitio en el que la naturaleza ha puesto las dos cosas, por lo tanto, similitud de propiedades».
} 
mentar la preeminencia del lugar teórico, el idealismo sobre la realidad, el método por encima del humanismo, la nula consideración de la memoria colectiva de los habitantes y su noción de espacio y ciudad. La anulación de estas características en favor de un espacio teórico devino en el espacio comprimido. Este modelo-pensamiento (con las connotaciones que asignó al contenido cerrado Riegl y luego fue retomado, desde el platonismo, por los arquitectos y urbanistas modernos) trajo consigo una ciudad segregada a niveles económicos (de propiedad privada), administrativos (de zonificación) o punitivos (el espacio disciplinar), que pusieron de manifiesto, con una visión idealista, ilustrada y completamente anti-cínica, la inconexibilidad del territorio.

La retícula define un archipiélago de «ciudades dentro de otras ciudades». Cuanto más exalta cada «isla»los valores distintos, más se refuerza la unidad del archipiélago como sistema. Puesto que el «cambio» está incluido en las «islas» que lo componen, ese sistema nunca tendrá que revisarse. En el archipiélago metropolitano cada rascacielos desarrolla su propio «folclore» instantáneo. Mediante la doble desconexión que implican la lobotomía y el cisma -separar la arquitectura exterior y la interior, y desarrollar esta última por pequeñas parcelas autónomas-, esas construcciones pueden dedicar sus exteriores sólo al formalismo, y sus interiores sólo al funcionalismo. De este modo no sólo resuelven para siempre el conflicto entre la forma y la función, sino que crean una ciudad donde unos monolitos permanentes exaltan la inestabilidad metropolitana. ${ }^{17}$

17. Koolhaas, 1994: 296

\section{REMISIÓN}

La forma en que la Modernidad se extingue y da paso a la Posmodernidad, y en que ésta se hace evidente, es sumamente sutil, sensiblemente «débil» en el sentido que le da Vattimo. Ya que la superación es una categoría típicamente moderna (en su lugar, hay una oposición débil, y una arquitectura débil, término del que se apropia Ignasí de Solá-Morales), la Modernidad no puede ser superada, como dijo Montaner, sino «remitida» como en Heiddeger: Ueberwindung, «remitir» en todas las acepciones del término, como remitir una carta o como la remisión de una enfermedad. ${ }^{18}$ Bajo esta condición, la Posmodernidad, o, para quien no quiera, la contemporaneidad es, más que un período, una actitud catártica y contestataria que explora nuevas formas de pensar el espacio.

Si la arquitectura se define como el arte del espacio, el estudio de éste debe ser vital, imprescindible y obligatorio para el arquitecto, más allá de una visión genérica, una abstracción geométrica o una generalización teórica. El espacio posmoderno inicia un proceso contracultural en todas direcciones, como oposición «débil» en el sentido que le da Vattimo al término y como emancipación de las formas disciplinarias descritas por Foucault, el filósofo del espacio. Su entendimiento y respectivos modelos históricos inician un proceso de liberación capaz de trazar nuevos diagramas (otra vez en el sentido foucaultiano, como mapas de poder) y otras dinámicas que no son ajenas ni a la vivencia de ciudad ni a la proyectación de la

18. Cf. Vattimo, 2000: 146 
arquitectura, como lo señalan las experiencias de ciudad y las tendencias de diseñadores y arquitectos contemporáneos. El desarrollo pragmático del espacio contemporáneo involucra tanto a fenómenos de un cinismo universal y difuso, como al modelo de «la ruptura de modelos», o sea, de la topología que vence a la tipología.

El espacio en la Posmodernidad tiende hacia la emancipación de las formas de control y los regímenes disciplinarios heredados de la llustración con su obsesión clasificatoria y sus principios de orden, novedad y progreso. El espacio posmoderno es por antonomasia transgresor, y por extensión, cínico, pues las características del cinismo están presentes en el momento de proyectar y vivirlo. En primera instancia, se ha pretendido en la sociedad liberal occidental (fuera de los regímenes absolutistas, dictatoriales y totalitarios) un espacio para los comunes, la democratización del territorio y el entendimiento e inserción del habitar en lo cotidiano. Esto se traduce desde el cinismo como la vida franca, que en la Posmodernidad supone una renuncia consciente a las formas de control derivadas de un espacio de poder sistemáticamente jerarquizado y dialéctico; se pierde la jerarquía del centro para rescatar no sólo la periferia, sino los puntos de conexión de la red, se privilegia (a veces violentamente) la transgresión y se vuelve a creer en la extensión por encima de la compresión. 


\section{BIBLIOGRAFÍA}

AU, S. (2012). Ballet and modern dance. Londres: Thames \& Hudson.

AUGÉ, M. (2000). Los «no lugares» espacios de anonimato. Barcelona: Gedisa.

BAUDRILLARD, J. (1978). Cultura y simulacro. Barcelona: Kairós.

DELEUZE, G. (1989). El pliegue, Leibniz y el barroco. Barcelona: Paidós.

DELEUZE, G. y GUATTARI, F. (2004). Mil mesetas. Capitalismo y esquizofrenia. Valencia: Pre-textos.

FARÍAS, C. (2003). Anatomía de una mente visionaria obsesionada con el presente: Rem Koolhaas. Tesis doctoral. México: unam.

FOUCAULT, M. (1968). Las palabras y las cosas. México: Siglo Veintiuno.

- (2010). El coraje de la verdad. Buenos Aires: Fondo de Cultura Económica.
- (2010b). «Los espacios otros», El cuerpo utópico (las heterotopías). Buenos Aires: Nueva Visión.

- (2013). Vigilar y castigar. México: Siglo Veintiuno.

KOOLHAAS, R. (1994). Delirious New York. usA: The Monacelli Press.

MONTANER, J. (2011). La modernidad superada. Barcelona: Gustavo Gili.

SLOTERDIJK, P. (2005). En el mundo interior del capital. Madrid: Siruela.

- (2007). Crítica de la razón cínica. Madrid: Siruela.

STEVEN, K. (1980). «Space and antispace», en Harvard Architectural Review, I.

TRICART, J. (1969). La epidermis de la tierra. Barcelona: Labor.

VATTIMO, G. (2000). El fin de la modernidad. Barcelona: Gedisa. 